\title{
BIAS CORRECTED PSD ESTIMATION WITH AN INTERFERENCE CANCELING ARRAY
}

\author{
Brian D. Jeffs and Karl F. Warnick \\ Department of Electrical and Computer Engineering, Brigham Young University \\ Provo, UT 84097, USA, bjeffs@ee.byu.edu, warnick@ee.byu.edu
}

\begin{abstract}
Adaptive interference canceling beamformers are known to suffer from beampattern distortion, particularly when interferers approach the nominal mainlobe. In some applications such as radio astronomy, even small pattern variations can be problematic. We address the issue of computing power spectral density (PSD) estimates at the output of a beamforming array in the presence of strong moving interference. A bias corrected PSD estimation algorithm is introduced which uses subspace projection methods to cancel interference at the beamformer. A correction step eliminates both patterndistortion-induced PSD bias and spatial response errors over the long-term PSD averaging window.
\end{abstract}

Index Terms - Adaptive arrays, Interference suppression, Radio Astronomy, Spectral analysis

\section{INTRODUCTION}

This paper considers the problem of computing power spectral density (PSD) estimates from the output of a beamforming array steered toward some signal of interest in the presence of strong moving interference. Conventional wisdom suggests using one of many candidate adaptive array processing algorithms to null out the interferer, followed by a separate PSD estimator which operates on the beamformer time series output. However, all known interference cancellation array processing algorithms introduce distortion in the effective beamforming spatial pattern, particularly as interference encroaches on the beam mainlobe. This biases the PSD estimate due to time varying changes in the beampattern.

In some applications, including radio astronomical (RA) observation, even modest beamshape distortions can be unacceptable. For example, in RA where signals of interest have power levels $30 \mathrm{~dB}$ or more below the noise floor, a small mainlobe distortion can corrupt sensitive calibrated measurements. Also, pattern variations in a radiometer system translate directly to an increase in the minimum detectable signal level since observing weak signals is only possible with long time integration and by subtracting a stable low variance estimate of the noise spectrum. Even variations in sidelobe pat-

This work was funded by National Science Foundation under grant number AST - 0352705 terns can make detection impossible due to induced variations in the observed noise levels. This is true even when beam response is held accurately to calibrated levels at the beam mainlobe center point. Thus for practical RA, beamshapes must be known precisely and must be be stable over time. This fact has hindered adoption of adaptive array interference cancellation techniques in RA even though man-made radio sources pose critical signal pollution problems. Typical RA interference comes from satellites or fixed ground transmitters, but due to Earth rotation and long integration times all appear to be moving relative to a deep space object of interest so that our assumption of moving interference is satisfied.

We introduce a new estimation algorithm which combines adaptive interference canceling with temporal PSD estimation and bias correction to remove the effect of beam distortions. This is possible if the signal of interest is stationary but interferers move during the PSD integration time window (which we will call the long term integration period, or LTI) . Though the LTI PSD estimate can be corrected for beam distortion bias, the method does not produce an unbiased beamformer time series output. The bias correction is based on the method of Leshem and van der Veen for array covariance estimation in a radio astronomical synthesis imaging [1]. We show how their technique can be adapted to PSD estimation.

\section{SIGNAL MODEL}

Consider a $P$ element sensor array which at time sample $n$ produces a length $P \times 1$ data vector

$$
\begin{aligned}
\mathbf{x}[n] & =\mathbf{a}_{s} s[n]+\mathbf{A}_{d}[n] \mathbf{D}[n]+\boldsymbol{\eta}[n] \\
\mathbf{D}[n] & =\operatorname{diag}\left\{\left[d_{1}[n], \cdots, d_{Q}[n]\right]\right\}
\end{aligned}
$$

where $s[n]$ is the signal of interest with corresponding array spatial response vector $\mathbf{a}_{s}, \boldsymbol{\eta}[n]$ is noise and $d_{q}[n]$ is one of $Q$ "detrimental" interfering sources whose corresponding array response vector forms the $q$-th column of $\mathbf{A}_{d}[n]$. Assume that $s[n]$ and $\boldsymbol{\eta}[n]$ are wide sense stationary random processes and $s[n]$ is spatially fixed relative to the array so that $\mathbf{a}_{s}$ is constant. On the other hand, both $\mathbf{A}_{d}[n]$ and $\mathbf{D}[n]$ are nonstationary over the long term due to interferer motion. However, since interference motion is relatively slow compared to the sample rate, over $L$ time samples called the "short term inte- 
gration (STI)" window length, $\mathbf{A}_{d}[n]$ is approximately constant and $\mathbf{D}[n]$ is approximately wide sense stationary.

It is desired to estimate the temporal PSD of $s[n]$, $S_{s}(\omega)=\mathcal{F}\left(E\left\{s[n+m] s^{*}[n]\right\}\right)$, without the corrupting effect of interferers $d_{q}[n]$ (here $\mathcal{F}$ is the Fourier transform with respect to $m, E\{\cdot\}$ is expectation, and * indicates complex conjugate). An obvious approach is to compute a beamformer steered to $s[n]$ followed by a sample PSD estimator. Let the beamformer output be $y[n]=\mathbf{w}_{j}^{H} \mathbf{x}[n]$ where $\mathbf{w}_{j}$ is a weight vector computed for the $j$-th STI to steer the beam's mainlobe maximum response toward $s[n]$ and to reduce sidelobe response in interferer directions. A simple spectral estimate is formed by applying the well known Welch's method [2] to $y[n]$, which can be expressed in the matrix-vector form

$$
\begin{aligned}
\hat{\mathbf{S}}_{y} & =\frac{\alpha}{M} \sum_{m=0}^{M-1}\left|\mathbf{F G} \mathbf{y}_{m}\right|^{\odot 2}, \quad \mathbf{G}=\operatorname{diag}\{\gamma\} \\
\mathbf{y}_{m} & =\left[y[m(N-O), \cdots, y[m(N-O)+N-1]]^{T}\right.
\end{aligned}
$$

where $N$ is the length of data window used in computing the discrete Fourier transform (DFT), $O$ is the number of overlapping samples in successive windows, $\gamma$ is the spectral shaping real time window (e.g. Hamming), $\mathbf{F}$ is the unitary DFT matrix, and $|\cdot|^{\odot 2}$ denotes element-wise magnitude squared. Averaging is computed over $M$ data windows, gross amplitude bias due to $\gamma$ is corrected by $\alpha=1 /\left(\gamma^{T} \gamma\right)$, elements of vector $\mathbf{S}_{y}$ correspond to frequency bins, and ' $\hat{.}$ ' indicates an estimated quantity. To the extent that $\mathbf{w}_{j}$ suppresses interferers in $y[n], \hat{\mathbf{S}}_{y}$ is a viable estimator for $\mathbf{S}_{s}=\left\{\left.S_{s}(\omega)\right|_{\omega=(2 \pi[0,1, \cdots, N-1]) / N}\right\}$.

For RA applications, noise power, even after beamforming, exceeds the desired signal. Thus $\mathbf{S}_{y, \eta} \gg \mathbf{S}_{y, s}$ element wise, where subscripts $\eta$ and $s$ denote noise and signal contributions to $\mathbf{S}_{y}$ respectively. In order to estimate $\mathbf{S}_{s}$, astronomers subtract a baseline noise reference estimate computed from a separate data set with matching statistics except for the absence of signal. For example, $\hat{\mathbf{S}}_{s}=\hat{\mathbf{S}}_{y}-\hat{\mathbf{S}}_{y, \eta}$ where $\hat{\mathbf{S}}_{y, \eta}$ is obtained by temporarily steering the beam away from the source and integrating over a very long LTI widow $(10 \mathrm{~s}$ to $10 \mathrm{~min}$.) to reduce estimation error variance. The signal spectrum can only be observed when sample error standard deviation in both $\hat{\mathbf{S}}_{y, \eta}$ and $\hat{\mathbf{S}}_{y}$ is below $\mathbf{S}_{s}$ in all frequency bins of interest, and noise statistics have not changed during either of the LTIs used to compute them. This requires exceptional beamformer stability such that variations in $\mathbf{w}_{j}$ over all $j$ in the LTI do not affect the noise spectral shape. Interferers must also be nulled in each STI to attenuate them well below the noise floor or they will dominate $\hat{\mathbf{S}}_{s}$.

We define the time dependent array autocorrelation matrix

$$
\begin{aligned}
\mathbf{R}_{x}[n] & =E\left\{\mathbf{x}[n] \mathbf{x}^{H}[n]\right\} \\
& =\sigma_{s}^{2} \mathbf{a}_{s} \mathbf{a}_{s}^{H}+\mathbf{A}_{d}[n] \boldsymbol{\Sigma}_{d}[n] \mathbf{A}_{d}^{H}[n]+\mathbf{R}_{\eta} \\
\boldsymbol{\Sigma}_{d}[n] & =\operatorname{diag}\left\{\left[\sigma_{d_{1}}^{2}[n], \cdots, \sigma_{d_{Q}}^{2}[n]\right]\right\}
\end{aligned}
$$

where ' $H$ ' is matrix complex conjugate and the $\sigma^{2}$ values represent the corresponding signal and interference variances. Note that only the interference terms in $\mathbf{R}_{x}[n]$ are time dependent, but due to approximate stationarity over an STI we may write

$$
\begin{aligned}
\mathbf{R}_{x}[n] & \approx \mathbf{R}_{x, j}, \forall j L \leq n<(j+1) L \\
\mathbf{R}_{x, j} & =\sigma_{s}^{2} \mathbf{a}_{s} \mathbf{a}_{s}^{H}+\mathbf{A}_{d, j} \boldsymbol{\Sigma}_{d, j} \mathbf{A}_{d, j}^{H}+\mathbf{R}_{\eta} \\
\mathbf{A}_{d, j} & =\mathbf{A}_{d}[j L], \quad \sigma_{d_{q}, j}^{2}=\sigma_{d_{1}}^{2}[j L]
\end{aligned}
$$

The STI sample estimator of $\mathbf{R}_{x, j}$ is computed as

$$
\begin{aligned}
\hat{\mathbf{R}}_{x, j} & =\frac{1}{L} \sum_{n=j L}^{(j+1) L-1} \mathbf{x}[n] \mathbf{x}^{H}[n]=\frac{1}{L} \mathbf{X}_{j} \mathbf{X}_{j}^{H} \\
\mathbf{X}_{j} & =[\mathbf{x}[j L], \mathbf{x}[j L+1], \cdots, \mathbf{x}[(j+1) L-1]] .
\end{aligned}
$$

\section{BIAS CORRECTED ARRAY PSD ESTIMATION}

The first order of business is to calculate beamformer weights, $\mathbf{w}_{j}$, using $\hat{\mathbf{R}}_{x, j}$ such that interferers will be cancelled while steering a high gain mainlobe toward $s[n]$. The beamformer must be updated each STI in order to track interferer motion and must satisfy

$$
\mathbf{w}_{j}^{H} \mathbf{A}_{d, j}=0 \text {, i.e. } \mathbf{w}_{j} \in \mathcal{N}\left\{\mathbf{A}_{d, j}\right\}
$$

at least approximately, where $\mathcal{N}\{\cdot\}$ indicates nullspace. Any number of algorithms can be considered, including linearly constraint minimum variance (LCMV), maximum SNR, and subspace projection spatial filtering $[1,3,4]$, but as mentioned, all will distort the quiescent (interference free) beampattern to some degree. A variety of methods have been proposed to control beam shape, including several mainlobe constraint schemes for LCMV and "diagonal loading" of $\hat{\mathbf{R}}_{x, j}$ to reduce sidelobe distortions in any of the algorithms [3]. None of these approaches can simultaneously achieve deep interference nulling and beamshape control in both mainlobe and sidelobes. The proposed method removes these bias effects in the PSD estimate to produce a distortionless effective beampattern over the LTI window.

We will use the subspace projection approach to form

$$
\mathbf{w}_{j}=\mathbf{P}_{j} \mathbf{w}
$$

were $\mathbf{w}$ is a deterministic fixed beamforming weight vector with the desired quiescent beam response and $\mathbf{P}_{j}$ is an estimate of $\mathbf{P}_{\mathbf{A}_{d, j}}^{\perp}$, the perpendicular subspace projection matrix for $\mathbf{A}_{d, j}$. Assuming interference dominates other signals and noise (the typical case in radio astronomy applications), one can use the partitioned SVD approach and let

$$
\mathbf{P}_{j}=\mathbf{I}-\mathbf{U}_{d} \mathbf{U}_{d}^{H}
$$

where $\mathbf{U}_{d}$ contains the first $Q$ ordered left singular vectors corresponding to the largest singular values in the decomposition $\hat{\mathbf{R}}_{x, j}=\mathbf{U} \Sigma^{\frac{1}{2}} \mathbf{V}^{H}, \mathbf{U}=\left[\mathbf{U}_{d} \mid \mathbf{U}_{s+\eta}\right]$. The number of 
independent interfering sources, $Q$, is either assumed known or is estimated using a method such as MDL [5].

When $O=0$ and $N=L$ so the FFT windows are nonoverlapping and match the STI length ${ }^{1}$, then exploiting symmetry of $\mathbf{G}$ and $\mathbf{F}$ and using (6) and (7) in (1) yields

$$
\hat{\mathbf{S}}_{y}^{T}=\frac{\alpha}{M} \sum_{j=0}^{M-1}\left|\mathbf{w}^{H} \mathbf{P}_{j} \mathbf{X}_{j} \mathbf{G F}\right|^{\odot 2} .
$$

Using properties presented in the Appendix for the matrix Kronecker product, $\otimes$, and column-wise Kronecker or KhatriRao product, $\circ,(8)$ can be expressed as

$$
\begin{aligned}
\hat{\mathbf{S}}_{y}^{T} & = \\
& \frac{\alpha}{M}\left(\mathbf{w}^{H} \otimes \mathbf{w}^{T}\right) \sum_{j=0}^{M-1}\left(\mathbf{P}_{j} \otimes \mathbf{P}_{j}^{*}\right)\left(\left(\mathbf{X}_{j} \mathbf{G F}\right) \circ\left(\mathbf{X}_{j} \mathbf{G F}\right)^{*}\right) .
\end{aligned}
$$

As in [1] define

$$
\mathbf{C}=\frac{1}{M} \sum_{j=0}^{M-1}\left(\mathbf{P}_{j} \otimes \mathbf{P}_{j}^{*}\right) .
$$

The final bias corrected array PSD estimator is then given by

$$
\begin{aligned}
\hat{\mathbf{S}}_{y, c}^{T}= & \frac{\alpha}{M}\left(\mathbf{w}^{H} \otimes \mathbf{w}^{T}\right) \mathbf{C}^{-1} \\
& \times \sum_{j=0}^{M-1}\left(\mathbf{P}_{j} \otimes \mathbf{P}_{j}^{*}\right)\left(\left(\mathbf{X}_{j} \mathbf{G F}\right) \circ\left(\mathbf{X}_{j} \mathbf{G F}\right)^{*}\right) .
\end{aligned}
$$

Computational and memory savings are possible for a practical implementation of (11) by using FFTs and deflating some of the Kronecker products.

Estimation bias is assessed by computing the expected value, where for simplicity we assume interferer motion is deterministic and $\mathbf{P}_{j}=\mathbf{P}_{\mathbf{A}_{d, j}}^{\perp}$ exactly. This implies $\mathbf{P}_{j}$ is not random, and insures the interference component in $\mathbf{X}_{j}$ is removed. Thus $\mathbf{X}_{j}$ may be replaced by $\mathbf{X}_{s+\eta, j}$ formed as in (6) but using only $\left(\mathbf{a}_{s} s[n]+\boldsymbol{\eta}[n]\right)$. For the proposed estimator

$$
\begin{aligned}
E\left\{\hat{\mathbf{S}}_{y, c}^{T}\right\}=\frac{\alpha}{M}\left(\mathbf{w}^{H} \otimes \mathbf{w}^{T}\right) \mathbf{C}^{-1}\left(\sum_{j=0}^{M-1}\left(\mathbf{P}_{j} \otimes \mathbf{P}_{j}^{*}\right)\right) \\
\times E\left\{\left(\mathbf{X}_{s+\eta, 1} \mathbf{G F}\right) \circ\left(\mathbf{X}_{s+\eta, s} \mathbf{G F}\right)^{*}\right\} \\
=\alpha\left(\mathbf{w}^{H} \otimes \mathbf{w}^{T}\right) E\left\{\left(\mathbf{X}_{s+\eta, 1} \mathbf{G F}\right) \circ\left(\mathbf{X}_{s+\eta, 1} \mathbf{G F}\right)^{*}\right\}
\end{aligned}
$$

where we have substituted (10) for $C$ and exploited the wide sense stationarity of $s[n]$ and $\boldsymbol{\eta}[n]$ so dependence of $\mathbf{X}_{s+\eta, j}$ on $j$ is removed under the expectation. This enabled factoring the expectation term out of the summation. The important points are that all spatial bias introduced by the time varying projection matrices $\mathbf{P}_{j}$ has been removed and that the bias corrected $E\left\{\hat{\mathbf{S}}_{y, c}^{T}\right\}$ has the same value as that of $E\left\{\hat{\mathbf{S}}_{y}^{T}\right\}$ obtained from a beamforming Welch's PSD estimator under conditions of no interference and fixed beamforming weights.

\footnotetext{
${ }^{1}$ We have developed a different formulation for overlapped arbitrary length windows, i.e. when $O \neq 0$ and $N \neq L$. This will be presented in a forthcoming paper.
}

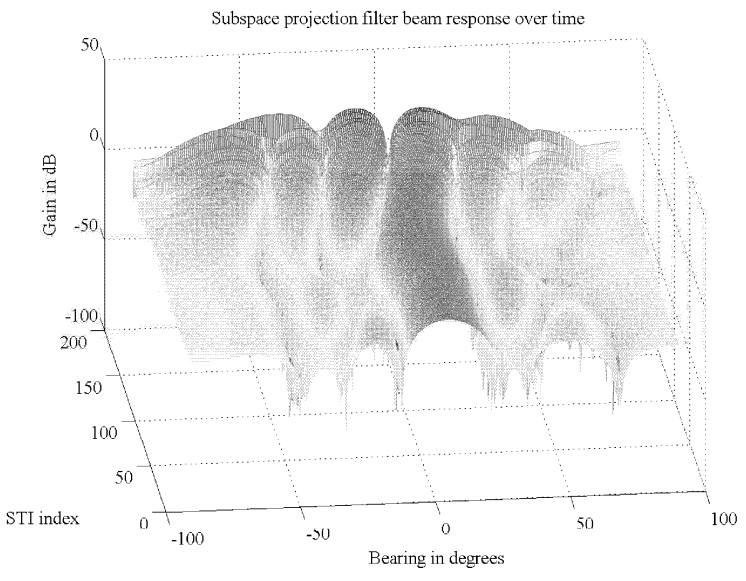

Fig. 1. Beampattern variation over time for each $\mathbf{w}_{j}$ in the LTI using subspace projection beamforming.

\section{SIMULATION RESULTS}

This section presents an experiment with a seven element half wavelength spaced uniform line array. The source of interest was at a bearing of $5^{\circ}$ with SNR of $-30 \mathrm{~dB}$. Two moving interferers were observed with initial bearings of $33^{\circ}$ and $-35^{\circ}$ and both moving at $5 \times 10^{-4}$ degrees per sample. The interference to noise ratio was $0 \mathrm{~dB}$ for each. The full LTI window includes $10^{5}$ samples, and $N=L=512$ samples with $O=0$. Noise was i.i.d. both spatially and temporally. The source and interferers were all narrowband at distinct frequencies so that their contributions to the PSD estimate could be readily identified.

Figure 1 presents the beamformer directional response pattern produced by $\mathbf{w}_{j}=\mathbf{P}_{j} \mathbf{w}$ for each STI, $j$. Note that two beamforming nulls track interferer motion. For the early STIs both interferers are in the sidelobes so the mainlobe steered to $5^{\circ}$ appears undistorted. However, in the later STIs the left-hand interferer encroaches on the mainlobe producing significant distortion and raising the sidelobe levels.

Figure 2 shows PSD estimates obtained using "conventional" fixed weights, w, and subspace projection weights, $\mathbf{w}_{j}$, with and without bias correction. The conventional beamformer was completely ineffective in suppressing interference. The curve for subspace projection without bias correction shows that interference was effectively excised, but due to beamshape distortions the noise floor is seen to rise. This would mask weak signals of interest and introduce instability in the noise baseline estimate. The bias corrected subspace projection PSD was computed using (11). The corresponding curve in Figure 2 shows the methods maintains the low noise floor of the fixed beamformer while effectively canceling interferers.

Figure 3 plots the "effective" beampattern of the three methods as seen through a PSD estimate over the full LTI window. Though for the subspace projection methods the 


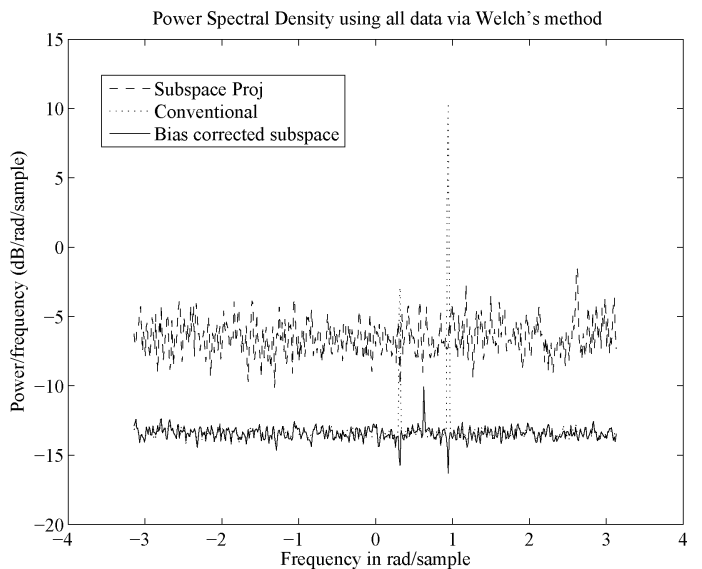

(a)

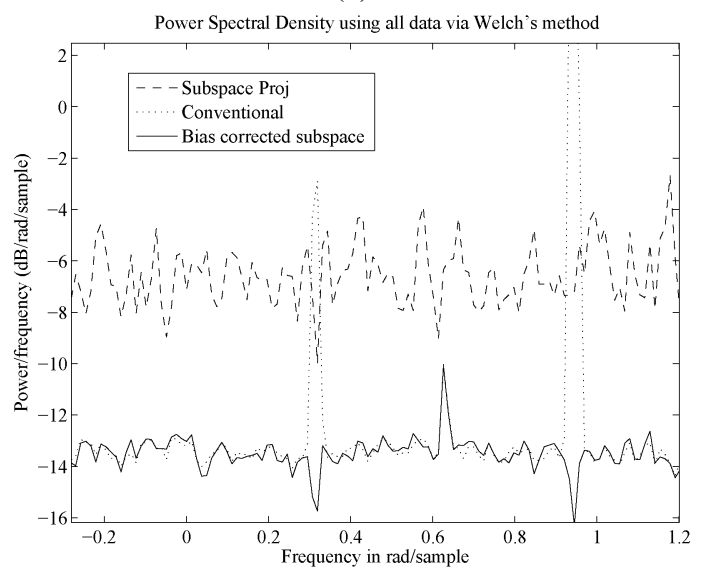

(b)

Fig. 2. PSD estimates over full LTI. (a) Signal of interest is at $0.62 \mathrm{rad}$ and interferers are at 0.3 and $0.9 \mathrm{rad}$. (b) Expanded view shows bias corrected PSD eliminates interference, accurately estimates signal level, and keeps noise floor low.

STI beampattern is changing over time, these curves represent the net effect as seen in the final PSD which used all time samples. Each point in the curves is obtained by placing a single source in the far field (with no noise or other signal). This probe signal is then processed for each STI using the corresponding $\mathbf{w}_{j}$ taken from the signal plus interference plus noise scenario. The probe signal level seen in the LTI PSD is interpreted as the effective beam response in the probe direction. The bias corrected subspace projection PSD has an effective beampattern indistinguishable from the quiescent pattern produced by $\mathrm{w}$ alone.

\section{CONCLUSIONS}

It has been shown that in the context of PSD estimation it is possible to use array processing techniques to cancel interference while maintaining a desired "effective" beampattern spatial response over the estimation time window. For very sen-

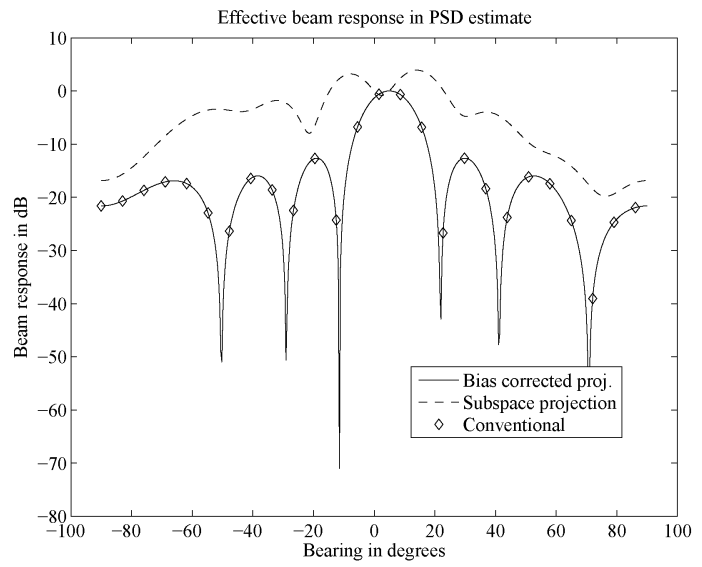

Fig. 3. Effective beam response patterns through the full LTI PSD estimates. Note the bias corrected subspace beamformer PSD has a completely undistorted effective beampattern matching the conventional quiescent response.

sitive calibrated detection and scientific measurement observations such as radio astronomy it is anticipated this method will help overcome the inertia which impedes adoption of critically needed interference canceling methods.

\section{APPENDIX}

The following matrix product properties were used in (9). For column vector $\mathrm{z}$ and arbitrary matrices $\mathbf{A}, \mathbf{B}, \mathbf{C}$ and $\mathbf{D}$ :

$$
\begin{aligned}
& \text { 1. }\left|\mathbf{z}^{T}\right|^{\odot 2}=\mathbf{z}^{T} \circ \mathbf{z}^{H} . \\
& \text { 2. }(\mathbf{A B}) \circ(\mathbf{C D})=(\mathbf{A} \otimes \mathbf{C})(\mathbf{B} \circ \mathbf{D}) . \\
& \text { 3. }(\mathbf{A B}) \otimes(\mathbf{C D})=(\mathbf{A} \otimes \mathbf{C})(\mathbf{B} \otimes \mathbf{D}) .
\end{aligned}
$$

\section{REFERENCES}

[1] A. Leshem and A.-J. van der Veen, "Radio-astronomical imaging in the presence of strong radio interference," IEEE Trans. Inform. Theory, vol. 46, no. 5, 2000.

[2] P.D Welch, "The use of the fast fourier transform for the extimation of power spectra," IEEE Trans. Audio Electroacoustics, vol. AU-15, pp. 70-73, June 1970.

[3] H.L. Van Trees, Detection, Estimation, and Modulation Theory, Part IV, Optimum Array Processing, John Wiley and Sons, 2002.

[4] S. Ellingson and G. Hampson, "A subspace-tracking approach to interference nulling for phased array-based radio telescopes," IEEE Trans. Ant. Prop., vol. 50, no. 1.

[5] M. Wax and T. Kailath, "Detection of signals by information theoretic criteria," IEEE Trans. on Acoustics, Speech, and Signal Processig, vol. ASSP-33, no. 2, Apr. 1985. 